\title{
Monitoring and Data Analytics for Optical Networking
}

\author{
Luis Velasco* and Marc Ruiz \\ Optical Communications Group (GCO), Universitat Politècnica de Catalunya (UPC), Barcelona, Spain \\ *e-mail:lvelasco@ac.upc.edu
}

\begin{abstract}
Increased levels of automation will be necessary in view of more stringent performance requirements that next generation optical transport networks need to support in a near term, not only for high capacity, but, even more importantly, for dynamicity, latency, and availability. All these aspects will become more relevant with the growing complexity of modern networks. Network automation targets resource re-optimization to rapidly adapt the network to the expected conditions, quick degradation detection to improve the quality of the connections, as well as failure detection and identification to facilitate maintenance. Network automation requires and implies the collection of data for performance monitoring, being then elaborated by data analytics algorithms to produce meaningful inputs for the network controller, which will finally program the underlying devices. In this paper, we analyze alternative architectures for monitoring and data analytics (MDA) and illustrative control loops are presented aiming at validating the usefulness of MDA to automate optical networks operation.
\end{abstract}

Keywords: Monitoring and data analytics, Autonomic optical networking

\section{INTRODUCTION}

After years of research and development, the Elastic Optical Networking (EON) technology is currently being deployed in optical transport networks. This technology enables among others: $i$ ) the capacity and / or reach increase of optical connections (hereafter, lightpaths) and ii) a finer and dynamic spectrum allocation. The first is enabled by the joint usage of coherent detection, advanced Digital Signal Processing (DSP) techniques, novel modulation formats and soft-decision Forward Error Correction (FEC) codes to recover Bit Error Rate (BER) within the Optical Transponders (TP). The second is possible thanks to programmable Wavelength Selective Switches (WSS) and Reconfigurable Optical Add-Drop Multiplexers (ROADM).

From a network control perspective, an enormous amount of research and standardization effort has been carried out, over the recent years, to implement the Software-Defined Networking (SDN) concept in optical networks. SDN relies on the separation of data and control planes, and leverages programmability and the usage of open interfaces. However, little to no attention has been paid to the operational loop (including monitoring, intelligence and management functionalities), relegating some of them into the Network Management Systems (NMS), and with limited practical operation capabilities. Although EON and SDN technologies can fulfill current capacity and dynamicity requirements, transport networks are expected to support the deployment of upcoming $5 \mathrm{G}$ mobile infrastructures in the near future; 5G mobile will extend far beyond previous generations and require an enhanced quality of experience for the final users with new services and improved network performance. However, given the increasing network complexity, the main challenge for operators will be to promptly respond to variable network conditions while ensuring full availability and optimization of network resources. Nonetheless, current optical networks are incorporating a complex ecosystem of devices and sensors, which will produce a large amount of data that can be exploited to optimize a network in real-time. To cope with such complex and timevariable scenarios, Machine Learning (ML) - based algorithms [1] are being proposed to facilitate the network operation and predictive maintenance. ML algorithms, fed with real measurements, are able to accurately estimate the Quality of Transmission (QoT) of new lightpaths, to anticipate capacity exhaustion and degradations, or to predict and localize failures, among others (see, e.g., [2]-[4]).

In this paper, we present several MDA architectures and highlight the pros and cons of each of them. Illustrative control loops are presented for three wide-scope use cases that require MDA-based solutions. The considered use cases bring a clear and complete vision of the validity and feasibility of MDA in the context of optical networks.

\section{MDA ARCHITECTURES}

In this section, we present and analyze architectural approaches to bring real MDA capabilities to the network.

The centralized architecture (Fig. 1a) consists in detaching the monitoring repository and the data analytics system, if any, from the NMS to create a separate specific centralized MDA controller that can interface the SDN controller and other systems within the control plane (see, e.g., Ciena Blue Planet). To keep the MDA architecture simple, let us consider that its only mission is to expose an interface to collect monitoring and telemetry data from the network devices. Measurements are stored in a (big data) repository, and data analytics algorithms can be devised to discover knowledge to be used to predict and/or to detect anomalies and degradations before they negatively impact on the network performance. Such predicted events can be notified to the SDN controller together and include a recommended action to guide the SDN controller; the recommended action is a suggestion that the SDN controller can follow or ignore and apply its own policies. As an example, in some cases BER degradation can be predicted ahead of time in a lightpath before any threshold is exceeded by analyzing the BER 
evolution as measured at the receiver; this is notified to the SDN controller together with a recommended action after analyzing several alternatives, including change of the modulation format (also via probabilistic shaping), reroute of the lightpath (e.g., to avoid some links); or also to increase, if possible, the amount of overhead used by the FEC. The notification to the SDN controller might trigger a re-configuration, hence closing the loop and adapting the network to the new conditions.
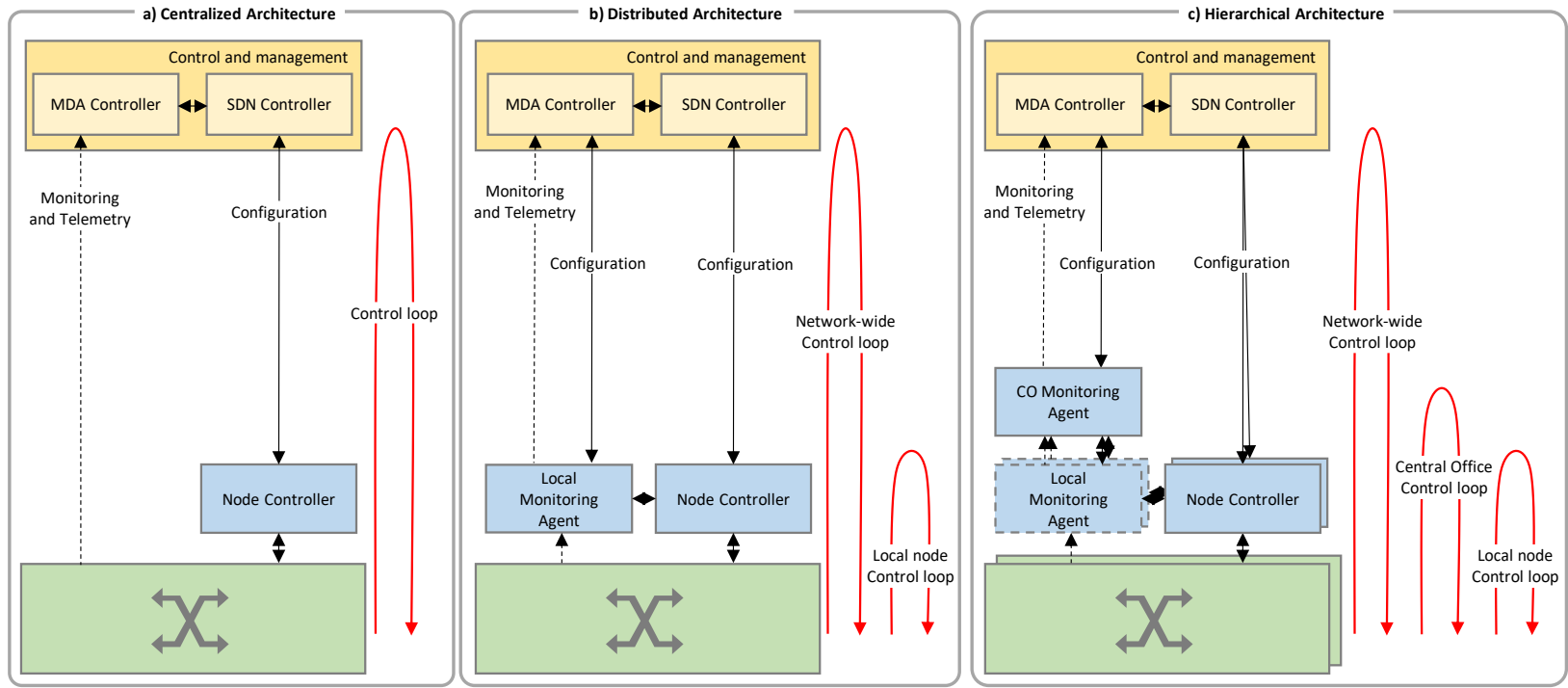

Fig. 1 Overview of the centralized (a), distributed (b), and hierarchical (c) MDA architectures.

The centralized MDA architecture presents some limitations; for instance, the time to detect an anomaly or degradation is related to the update frequency. Therefore, to reduce the detection times, the amount of data to be conveyed to the MDA controller needs to be increased accordingly. Another issue is related to the control of monitoring; specifically, to activate telemetry on-demand once an event has been detected. To overcome these problems, the distributed architecture (Fig. 1b) includes MDA agents in charge of collecting measurements from a single node, while keeping the MDA controller centralized [6]-[7].

The MDA agent exposes two unified interfaces toward the MDA controller for collecting data and monitoring configuration; in addition, specific interfaces for data collection and monitoring control allow the MDA agent connecting with the network device. The data analytics capabilities deployed close to the network nodes enable local control loops implementation; measurements can be analyzed locally, and configuration can be tuned and adapted to changing conditions. However, the co-existence of two controllers, the SDN and the MDA, in charge of configuring network devices, might create conflicts, so it would be desirable to clearly separate responsibilities among them.

The distributed architecture includes a dedicated MDA agent for every node, which might present some limitations when disaggregated optical network nodes and monitoring devices are deployed within the same central office (CO) [7]. For this reason, the hierarchical architecture (Fig. 1c) includes a per-CO MDA agent that collects measurements from every network device in the $\mathrm{CO}$ and exposes a single set of interfaces toward the MDA controller; measurements from one device can be analyzed in the CO MDA agent and configuration can be tuned to another device within the same $\mathrm{CO}$, thus minimizing the intervention of the MDA controller.

\section{ILLUSTRATIVE CONTROL LOOP IMPLEMENTATION}

This section illustrates how three illustrative use cases can be implemented. To this end, let us assume a disaggregated scenario, where COs are equipped with TP nodes and ROADMs and the hierarchical MDA architecture is selected. Apart from the MDA, the control plane includes an SDN controller in charge of configuring the optical network, a planning tool running optimization algorithms for provisioning and in-operation network planning purposes [8], and an NMS for human operators to manage the network.

Additionally, it is worth highlighting that if external systems, such as planning tools, require to access data in the MDA controller upon request, it is necessary to define an additional interface; the data that are available, as part of the MDA, are not only raw measurements coming from the network devices, but also estimated data generated by ML algorithms.

\subsection{Lightpath provisioning with a reduced margin}

In this first use case, we focus on the provisioning of lightpaths minimizing the system margin, which can be derived from the OSNR and / or the TP's pre-FEC BER threshold according to the transmission scenario. OSNR estimation at optimal lunch power and in nonlinear regime requires data from monitoring the optical channel and the devices configuration, which we assume that are already available in the MDA controller (labeled 0 in 

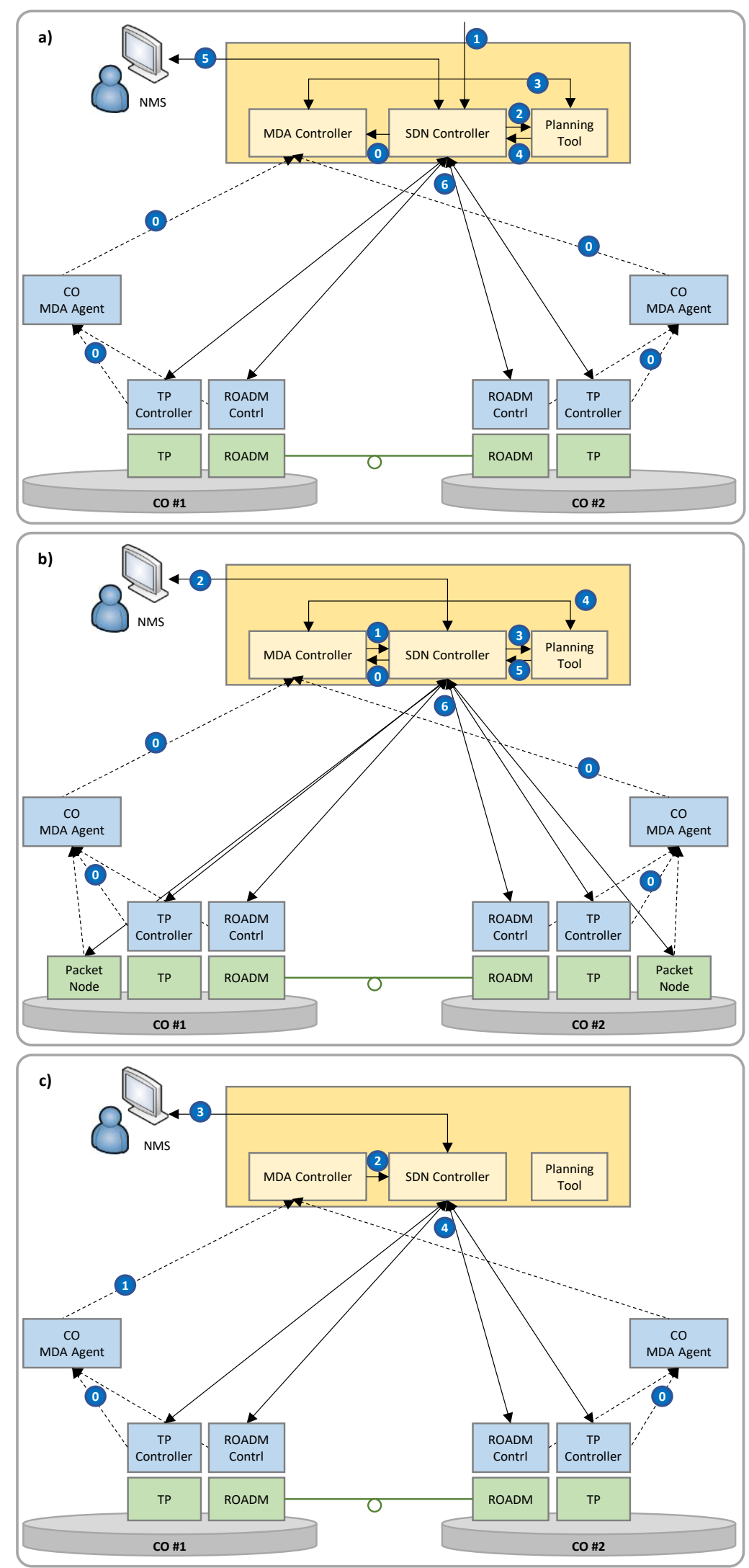

Fig. 2 Control loops implementation: Lightpath provisioning with reduced margin $(a)$, dynamic network adaptation $(b)$, and lightpath degradation detection and modulation format adaptation (c).
Fig. 2a). Besides this information, also parameters related to network infrastructures (such as fiber types and link lengths) are required. These might be collected, e.g., from the SDN controller, the NMS and inventory systems.

When a lightpath set-up request arrives at the SDN controller (1), the latter relies on the planning tool for the computation of the route, spectrum allocation, modulation format and other parameters that contribute to minimize the system margin while guaranteeing its QoT (2). To compute the optimal solution, the planning tool needs to access data from the MDA controller (3); once a solution has been found, it is sent back to the SDN controller (4).

Here, three possibilities might exist: $i)$ the lightpath can be established, and an optimal configuration has been found; $i$ ) the lightpath can be established provided that the configuration of other lightpaths is first changed; and iii) no solution has been found. In the second case, the planning tool returns the optimal configuration found for the requested lightpath, together with a (reactive) recommended action for the SDN controller to modify the configuration of a subset of already established lightpaths; in this case, the SDN controller might request the human operator to confirm the re-configuration through the NMS (5). Finally, in the case that the lightpath can be established with or without network re-configuration, the SDN controller configures the network devices accordingly. Further optimization can then be achieved by observing the QoT of each established lightpath, aiming at identifying possible transmission adaptions (e.g., FEC, modulation format) leading to margin reductions closer to the predefined target values.

\subsection{Dynamic network adaptation}

In the previous control loop,

the planning tool issued a recommended action for re-configuration because of a previous request from the SDN controller (named reactive). In this and the next use cases, the MDA controller will issue recommendations to the 
SDN controller as a result of observing what is happening in the network and aiming at anticipating the most relevant events. In this context, we refer them to as proactive recommendations.

As before, we assume that data are already available within the MDA controller (labeled 0 in Fig. 2b). ML algorithms running in the MDA controller can use the measured packet traffic volume to determine a traffic model for the traffic between every origin and destination CO. Such traffic models can be used to compare the expected traffic against the provisioned capacity and therefore, when the measured or the expected traffic for the near future is close to the allocated capacity, the MDA controller issues a notification to the SDN controller including a recommended action to reconfigure the allocated capacity (1).

In such case, the SDN controller might inform the human operator through the NMS (2) and then, request the planning tool to compute the optimal capacity configuration for the detected event (3). For such computation, the planning tool needs data from the MDA controller, e.g., the expected traffic matrix, e.g., for the next hours (4) [2]. Such traffic matrix can be computed assuming the the $95^{\text {th }}$ percentile traffic volume expected for every origindestination pair. With such a traffic matrix, an optimization algorithm running in the planning tool can compute the optical capacity allocation and respond to the SDN controller (5). Finally, the SDN controller implements the re-configuration in the network (6).

\subsection{Lightpath degradation detection and modulation format adaptation}

In this use case, let us imagine an already established lightpath being monitored, where BER measurements are collected by the MDA agents connected to the end TPs (labeled 0 in Fig. 2c). A data analytics algorithm running within the MDA agents can be in charge of detecting BER trends to anticipate QoT degradation [3]. In case of QoT degradation detection, a decision can be locally made without the intervention of the MDA controller. For instance, modern TPs are capable of identifying the modulation format of the received signal by means of DSP. Therefore, a change in the modulation format employed for a lightpath can be initiated in one of the transmitters and the end TPs will automatically realize of such change and carry out the same in the opposite direction (such local control loop is not shown in Fig. 2c). However, in disaggregated multi-vendor scenarios, both ends could need to be simultaneously re-configured. To that end, the MDA agent sends a notification to the MDA controller (1) that evaluates the capabilities of both TPs and evaluates the possibilities. The degradation detection together with a recommendation (e.g., change the modulation format to a more robust one) is sent to the SDN controller (3) that implements it in the devices, might be after checking it with the operator in the NMS (3-4).

\section{SUMMARY}

The role of MDA in optical networking has been studied through three wide-scope use cases covering the main network operations: $i$ ) network planning and provisioning, ii) dynamic network adaptation, and iii) degradation detection and failure localization, where clear benefits have been unveiled. Illustrative control loops supporting examples of the selected use cases have been shown. As a final remark, although the technologies supporting MDA in optical networks are ready, there is still a significant amount of discussion required within the relevant standardization forums and industrial Open Source projects, to leverage this work fully.

\section{ACKNOWLEDGEMENTS}

The research leading to these results has received funding from the EC through the METRO-HAUL project (G.A. $\mathrm{n}^{\mathrm{o}}$ 761727), from the Spanish MINECO TWINS project (TEC2017-90097-R), and from the Catalan Institution for Research and Advanced Studies (ICREA).

\section{REFERENCES}

[1] D. Rafique and L. Velasco, "Machine Learning for Optical Network Automation: Overview, Architecture and Applications [Invited Tutorial]," IEEE/OSA Journal of of Optical Comm. and Networking, vol. 10, pp. D126-D143, 2018.

[2] F. Morales, et al., "Virtual Network Topology Adaptability based on Data Analytics for Traffic Prediction," IEEE/OSA Journal of Optical Comm. and Networking, vol. 9, pp. A35-A45, 2017.

[3] A. P. Vela et al., "BER Degradation Detection and Failure Identification in Elastic Optical Networks," IEEE/OSA Journal of Lightwave Technology, vol. 35, pp. 4595-4604, 2017.

[4] A. P. Vela et al., "Soft Failure Localization during Commissioning Testing and Lightpath Operation," IEEE/OSA Journal of Optical Comm. and Networking, vol. 10, pp. A27-A36, 2018.

[5] Ll. Gifre et al., "Autonomic Disaggregated Multilayer Networking," IEEE/OSA Journal of Optical Comm. and Networking, vol. 10, pp. 482-492, 2018.

[6] L. Velasco et al., "An Architecture to Support Autonomic Slice Networking [Invited]," IEEE/OSA Journal of Lightwave Technology, vol. 36, pp. 135-141, 2018.

[7] L. Velasco et al., "Building Autonomic Optical Whitebox-based Networks," IEEE/OSA Journal of Lightwave Technology, vol. 36, pp. 3097-3104, 2018.

[8] L. Velasco and M. Ruiz, Provisioning, Recovery and In-operation Planning in Elastic Optical Networks, Wiley, 2017. 